\title{
Implementación de una estrategia virtual de aprendizaje y el logro de competencias en el estudiante universitario
}

\author{
Implementation of a virtual learning strategy and the achievement of competences in \\ the university student
}
Implementação de uma estratégia de aprendizagem virtual e obtenção de competências no estudante universitário

ARTÍCULO DE REVISIÓN

\author{
Wendy Del Carmen Carpio Vásquez \\ wencv4@gmail.com \\ https://orcid.org/0000-0003-4702-837X \\ Universidad César Vallejo, Chiclayo-Perú
}

\author{
Julio César Arana Delgado \\ aranajuliomd@gmail.com \\ https://orcid.org/0000-0002-6273-6334
}

Universidad César Vallejo, Chiclayo-Perú

Recibido 08 de enero 2021 | Arbitrado y aceptado 15 de febrero 2021 | Publicado en 01 abril 2021

\begin{abstract}
RESUMEN
El presente artículo tiene como objetivo general destacar las ventajas del uso de estrategias virtuales de aprendizaje, con énfasis en el Foro Virtual, estudiando su impacto en el logro de competencias en la educación universitaria, además del desarrollo de habilidades y capacidades a partir de su utilización. La metodología utilizada se basa en un diseño no experimental a través de una revisión sistemática con un método descriptivo analítico. Como conclusión, queda establecido la importancia y el impacto positivo de los foros de discusión como herramienta para mejorar el proceso de enseñanza-aprendizaje, su utilidad para la discusión de contenidos y para implantar un proceso de aprendizaje activo y participativo de los estudiantes.; además, el desarrollo de habilidades inferenciales, pensamiento y juicio crítico son elementos adicionales trascendentes para la evolución del proceso educativo, donde los estudiantes participan de manera activa y aportan valor pedagógico al proceso educativo.
\end{abstract}

Palabras clave: Foro Virtual; competencias; aprendizaje; pensamiento crítico

\section{ABSTRACT}

The general objective of this article is to highlight the advantages of the use of virtual learning strategies, with emphasis on the Virtual Forum, studying its impact on the achievement of competencies in university education, in addition to the development of skills and abilities from its use. The methodology used is based on a non-experimental design through a systematic review with a descriptive analytical method. In conclusion, it is established the importance and positive impact of discussion forums as a tool to improve the teaching-learning process, its usefulness for content discussion and to implement an active and participatory learning process for students. In addition, the development of inferential skills, thinking and critical judgment are additional transcendent elements for the evolution of the educational process, where students participate actively and contribute pedagogical value to the educational process.

Key words: Virtual Forum; skills; learning; critical thinking

\section{RESUMO}

O objetivo geral deste artigo é destacar as vantagens da utilização de estratégias de aprendizagem virtual, com ênfase no Fórum Virtual, estudando seu impacto no alcance de competências na formação universitária, além do desenvolvimento de competências e habilidades a partir de sua utilização. A metodologia utilizada é baseada em um desenho não experimental por meio de uma revisão sistemática com método analítico descritivo. Em conclusão, estabelece-se a importância e o impacto positivo dos fóruns de discussão como ferramenta para aprimorar o processo ensinoaprendizagem, sua utilidade para a discussão de conteúdos e para implementar um processo de aprendizagem ativo e participativo para os alunos. Além disso, o desenvolvimento de habilidades inferenciais, pensamento e julgamento crítico são elementos transcendentes adicionais para a evolução do processo educacional, onde os alunos participam ativamente e contribuem com valor pedagógico para o processo educacional.

Palavras-chave: Fórum virtual; Competências; Aprendendo; Pensamento critico 


\section{INTRODUCCIÓN}

El logro de competencias del estudiante universitario, se materializa en un conjunto de asignaturas que tienen como objetivos desarrollar en el estudiante una serie de capacidades requeridas por las exigencias de la sociedad en la que este se desenvolverá y cuyo desarrollo puede ser demostrado. Este enfoque tiene una serie de ventajas importantes para la educación superior, en particular en aquellas carreras como las ingenierías que tienen un compromiso formativo centrado en el dominio de procedimientos. Lo anterior se debe a que, el enfoque de competencias permite expresar mejor las capacidades que deben disponer los egresados al momento de completar sus estudios. De esta manera se facilita el proceso de transición entre el término de los estudios y la incorporación al mercado laboral. (Icarte y Labate, 2016).

El rendimiento académico del estudiante universitario, como indicador de eficiencia y calidad de la educación superior, constituye una preocupación constante para las instituciones a nivel mundial, por lo que desde hace cinco décadas ha sido un tema relevante dentro de la investigación a fin de explicarlo y dar respuesta a las problemáticas asociadas, tales como el abandono, rezago y reprobación. Sin embargo, dada su complejidad, derivada de los múltiples factores y variables que inciden en este, la consulta de investigaciones sobre el tema arroja un gran número de trabajos abordados desde diferentes perspectivas que coinciden en la relevancia de la temática, pero sobre todo en la falta de soluciones que permitan prevenir y disminuir los problemas relacionados con el rendimiento académico y contribuir a su mejora. (Ordaz y García, 2018).

En el contexto educativo a nivel superior, suele hacerse referencia a la continua necesidad de promover nuevas formas de aprender, existiendo de alguna forma consenso de que para ello habrá que proponerse procedimientos, metodologías y modelos que aprovechen los diferentes recursos que suministra la tecnología y propiamente aquellas que están relacionadas con los entornos virtuales de aprendizajes. Siendo esto así, también hay que plantearse la necesidad de proveer al estudiante recursos que puedan ser empáticos cognitivamente con ellos, tal como lo afirma La Madriz, (2016) en su estudio Factores que promueven la deserción del aula virtual, donde encontraron que para reducir la deserción hay que enfrentar y evitar el sentimiento de frustración que puede concebir el estudiante con los inconvenientes en el uso del entorno virtual, en los componentes personales, técnicos, académicos o económicos.

El bajo rendimiento y la consecuente deserción académica son problemas crecientes que pueden afectar la situación socioeconómica de un país. Se han postulado los estilos de aprendizaje como factores determinantes del rendimiento académico y es necesario estudiarlos, con el fin de proponer estrategias encaminadas a su resolución. Un estudio realizado en estudiantes de Medicina en Colombia reportó que el rendimiento académico en los estudiantes de medicina puede estar influenciado por otras variables diferentes al estilo de aprendizaje. Sin embargo, se deben tener en cuenta las diversas limitaciones técnicas que este estudio pudo haber tenido. (Merchán, Saavedra, García, Ospina, Aragón, y Martínez, 2017).

La generación de espacios de interacción y la disponibilidad de los estudiantes posibilita que los procesos formativos en modalidad a distancia sean más satisfactorios para éstos y el docente, siempre y cuando la interacción sea 
frecuente y asertiva, como lo demuestra, Moncayo, Pereira, y Luján, (2018), quienes realizaron un análisis de la participación de los estudiantes en actividades síncronas y asíncronas en estudiantes universitarios y la incidencia en su rendimiento académico, empleando dentro de las actividades asíncronas el foro académico virtual , encontrando que el foro es la actividad de mayor participación, en tanto que el chat y la video colaboración ocupan el segundo y tercer lugar, respectivamente. Este resultado guarda relación con otros trabajos y artículos revisados, en los que también la actividad asincrónica es la más utilizada por el estudiante, aspecto que obedecería al tiempo de apertura y desarrollo de la actividad.

Benavides, Villacís y Ramos, (2017) realizaron un estudio sobre el nivel de desarrollo cognitivo en los estudiantes por la utilización del entorno virtual de aprendizaje, concluyendo que los resultados alcanzados determinan la necesidad de que los alumnos y docentes adquieran una mejor comprensión sobre el funcionamiento de un espacio virtual de aprendizaje, y de cómo generar con una estrategia virtual de aprendizaje actividades de enseñanza-aprendizaje para generar conocimiento. Los directivos de la institución, deben hacer los correctivos pertinentes $\mathrm{y}$ eficaces para el mejoramiento de la calidad del proceso de enseñanza-aprendizaje con apoyo virtual, ya que existen evidencias de uso de actividades que permiten adquirir conocimientos.

Del mismo modo, Soto, Torres y Zamudio (2018) resaltaron una aceptación favorable para este tipo de actividades, por lo que se concluye que los ambientes virtuales de aprendizaje resultan favorables para quienes tienen un horario laboral que les impide estudiar en la modalidad presencial. Asimismo, las didácticas aplicadas en el aprendizaje en línea resultan favorables para el desarrollo de habilidades de colaboración. Asimismo, concluyeron que los espacios virtuales de aprendizaje propician la construcción colectiva del conocimiento al presentar didácticas adecuadas para ello, lo que fortalece la habilidad de la colaboración en línea.

En este sentido, los foros académicos virtuales son los ambientes propicios para la generación de conocimiento entre todos los que participan en ellos. Por lo cual, son definidos en la guía del estudiante de la Universidad Señor de Sipán (USS, 2014) como herramientas que propician la participación a través de dinámicas de debate que, si bien se valen de los argumentos y reflexiones planteadas por los estudiantes (para lo cual se han informado del punto de discusión y plantean un juicio crítico), deben conducir hacia un diálogo positivo donde la verdadera finalidad sea indagar y utilizar el diálogo para el intercambio de conocimientos, ideas y enfoques variados sobre el tema que se esté discutiendo. Igualmente, los foros académicos se clasifican en foros de socialización, debate y argumentación y en ellos deben quedar expresadas las elaboraciones que conducen al conocimiento. Estos foros deben admitir y promover la expresión de inquietudes que permitan identificar y proponer categorías para la discusión, valorando o acreditando diferentes propuestas, con el fin de fortalecer y fomentar la capacidad argumentativa $y$, sobretodo; reflexiva de los participantes.

El foro de discusión, en el ámbito de la formación profesional, se basa en una comunicación textual que permite el despliegue de una serie de recursos para relacionarse con los pares a través del lenguaje escrito. Así, los participantes manifiestan su conocimiento disciplinario de un tema, las 
actitudes frente al mismo y a los compañeros, así como las habilidades para externar el pensamiento. Sin embargo, dar seguimiento al empleo de estos recursos implica adoptar formas innovadoras de evaluación que den cuenta del uso integral de los recursos para identificar la manifestación de la competencia argumentativa en un foro de discusión. (Guzmán y Flores, 2020).

Asimismo, Castro, Suárez, y Soto, (2016), Estudiaron el aporte de los foros virtuales como instrumentos de evaluación para desarrollar habilidades de autorregulación y conocer la valoración de los estudiantes y docentes sobre el uso de esta metodología; encontrando cambios en las creencias y en el uso de las habilidades cognitivas de pensamiento crítico de los alumnos, que reflejan el inicio de un proceso de desarrollo de habilidades sociales y cognitivas; concluyendo que el uso de foros virtuales como metodología de evaluación es adecuado, porque, además de medir los aprendizajes propios de una unidad de contenidos, favorece el desarrollo de habilidades cognitivas y sociales y los alumnos la perciben como una dinámica más entretenida y motivadora.

En consecuencia, la presente investigación pretende dilucidar el uso de estrategias virtuales de aprendizaje, con énfasis en el Foro Virtual de debate y su impacto en el logro de competencias en los estudiantes universitarios, teniendo en cuenta que las Tecnologías de la Información y Comunicación (TIC), ya cuentan un periodo significativo de difusión y uso como herramienta innovadora que optimiza el logro de competencias y el aprendizaje significativo y que su auge e influencia han crecido exponencialmente en el presente año debido a la disposición del Ministerio de educación de suspender la prestación presencial del servicio educativo mientras esté vigente el estado de emergencia nacional para la prevención y control de COVID19. Con lo cual crece la necesidad de implementar diversas estrategias didácticas innovadoras que permitan fomentar un proceso cognitivo dinámico, priorizando un aprendizaje significativo y autónomo que alcance los propósitos de formación que exige el mundo de hoy. (MINEDU, 2020).

Por lo tanto, el objetivo general de la investigación es destacar las ventajas del uso de estrategias virtuales de aprendizaje, con énfasis en el Foro Virtual, estudiando su impacto en el logro de competencias en la educación universitaria, además del desarrollo de habilidades y capacidades a partir de su utilización. Para lograr este cometido es necesario plantear la siguiente pregunta: ¿la implementación de una nueva estrategia virtual de aprendizaje podrá lograr generar nuevas competencias en el estudiante universitario y ayudar a mejorar el rendimiento académico? La justificación del trabajo se basa en el descubrimiento de nuevas herramientas tecnológicas de interacción entre personas que permitan el debate de ideas y a su vez generen el conocimiento necesario para cambiar el rumbo del rendimiento académico de los estudiantes universitarios, así como el fortalecimiento de los espacios virtuales de enseñanza-aprendizaje a nivel de la educación superior.

\section{METODOLOGÍA}

La presente investigación posee un nivel descriptivo cualitativo. Esta investigación consistió en una revisión sistemática de artículos científicos indexados en cinco bases de datos científicas con verificable rigor académico en ciencias psicológicas y ciencias de la educación; esta fueron: Scopus, Scielo, Web of Science, Google Scholar; y Elsevier, se 
concreta exclusivamente en la recopilación de información de diversas fuentes, con el objeto de organizarla describirla e interpretarla de acuerdo con ciertos procedimientos que garanticen confiabilidad y objetividad en la presentación de los resultados (Palella \& Martins, 2010).

Para el desarrollo de la búsqueda se plantearon palabras claves como "competencias estudiantiles", "rendimiento académico", "TIC", "educación virtual", "estrategias virtuales" y "foro virtual", además de tomar en cuenta referencias no mayor a 5 años. La búsqueda arroja 19 referencias bibliográficas que cumplen con la necesidad de garantizar el objetivo general de la investigación, las cuales se organizaron a razón del desarrollo de la misma, siguiendo hilo descriptivo-analítico, identificando y revisando artículo por artículo, por medio de la lectura e identificación de estrategias y dimensiones manejadas por los autores, considerando al mismo tiempo una visión deductiva, partiendo de la generalidad del contenido del artículo y llegando a lo específico que fue el contenido relevante para ser tenido en cuenta en esta investigación.

El público objetivo para la presente investigación corresponde a los profesionales que laboran como docentes en educación superior universitaria, con la finalidad de implementar el foro virtual de debate como estrategia de aprendizaje para facilitar el logro de competencias y rendimiento académico en sus estudiantes en el contexto de una educación virtual.

\section{RESULTADOS Y DISCUSIÓN}

A través de diversos estudios cualitativos se ha confirmado la importancia de conocer el adecuado manejo de las herramientas virtuales, con el propósito de un mejor aprovechamiento de los estudiantes universitarios tal como lo argumenta Díaz, (2018) su estudio posee un cualitativo etnográfico para identificar los conocimientos, actitudes y prácticas del uso de redes sociales digitales y su rol en la salud y socialización de los estudiantes de medicina. De acuerdo con la información recolectada y procesada se logró definir a las redes sociales como herramientas de comunicación e información en la actualidad, creadas como plataformas para mantener el contacto entre personas. Sin embargo, la información dispuesta en la red es diversa y su credibilidad es dudosa por lo que requiere una fuente segura. Su accesibilidad ha sido factible gracias a la aparición y extensión de las redes de internet y la tecnología, principalmente computadoras y celulares inteligentes, que la mayoría de la población lo posee. Asimismo, los intercambios a nivel de redes sociales han sido considerados como oportunidades laborales rápidas y que se las puede realizar desde la comodidad de sus hogares.

La influencia del uso de foros virtuales resulta una metodología positiva para optimizar el rendimiento académico, este postulado se corrobora con diversas investigaciones como Galindo, De la Torre, y Vera, (2020), quienes estudiaron la influencia del uso de un entorno virtual en el rendimiento académico de estudiantes de odontología en Cusco en el presente año, utilizó el Moodle: Modular Object-Oriented Dynamic Learning Environment (Entorno modular de Aprendizaje Dinámico Orientado a Objetos) aplicado al grupo experimental, para luego medir el rendimiento académico en ambos grupos mediante cuestionario de preguntas cerradas y abiertas que se aplicaron al finalizar cada periodo académico. El rendimiento académico, medido en esos términos, fue 
significativamente mayor en los estudiantes que pertenecen al grupo experimental con un promedio de 15.17, frente al rendimiento del grupo control que tuvo un promedio de 11.19. Por lo tanto, se concluyó que la influencia del uso del entorno virtual en el rendimiento académico de los estudiantes de grupo experimental fue positiva. (Galindo et al. 2020).

En el mismo sentido, Villalobos (2018) investigó acerca del efecto de las aulas virtuales en el aprendizaje por competencias en los estudiantes del Instituto Superior Cueto Fernandini en Comas, con el uso de esta estrategia en el grupo experimental para posteriormente ver su efecto en el aprendizaje por competencias. Se administró un pre test a ambos grupos de control y de experimentación, el grupo de experimentación recibió el tratamiento experimental y el otro no (grupo de control). Posteriormente los grupos son comparados en el post test para analizar si el tratamiento experimental tuvo un efecto sobre la variable dependiente. Los resultados en el post test indican una diferencia de medias de 6 puntos a favor del grupo experimental, es decir el promedio de notas fue significativo en el post prueba en todas las dimensiones del aprendizaje por competencias.

En una interesante investigación; Navarro, Cragno y Di Bernardo (2016), tratan sobre: Analítica del Aprendizaje aplicada a los estudiantes de Medicina en Foros de discusión de casos clínicos en Medicina III. Dentro de sus conclusiones observaron que: en la construcción de nuevos entornos de aprendizaje en línea, donde los estudiantes aprenden desempeñándose por medio de la ayuda de la red social en un foro virtual. En el resultado de la presente investigación se muestra que, los estudiantes han considerado que participar en ellos los ha ayudado a trabajar en equipo, reflexionar con sus compañeros y relacionar conocimientos previos por lo cual parece de gran relevancia poder desarrollar la analítica del aprendizaje, que permita conocer el comportamiento de profesores y estudiantes, y así, evidenciar los problemas en este ámbito y generar las mejoras. La analítica del aprendizaje del comportamiento de los estudiantes en herramientas virtuales de aprendizaje colaborativo, provee insumos relevantes para el diseño de los cursos modificando el perfil social virtual del alumnado.

En otro estudios cualitativos se recomienda el uso del foro virtual, por ejemplo lo investigado por Kutugata, (2016) que trata sobre: Foros de discusión: herramienta para incrementar el pensamiento crítico en educación superior. Se encontró que: es recomendable la práctica de más foros de discusión en los cuales el participante tenga la necesidad de debatir con mayor profundidad y evaluar sus réplicas y contrarréplicas. Es con este ejercicio de retroalimentación, que se eleva la calidad de sus aportaciones. Por lo tanto, es conveniente un mayor número de foros de discusión en diversos cursos académicos que fomenten la participación fundamentada en dichos debates, así como la habilidad de argumentar conceptos académicos e incorporar conocimientos previos con la nueva información adquirida. Además, como todo proceso de aprendizaje, el lograr incrementar los niveles de pensamiento crítico es cuestión de práctica en aportaciones significativas en foros de discusión con la fundamentación pertinente y la vinculación a conocimiento previo o externo al adquirido en el curso en cuestión. El incorporar recursos académicos diversos que enriquezcan el proceso de enseñanza-aprendizaje en entornos 
virtuales, tanto en cursos presenciales como en semipresenciales y en línea, ayuda a alcanzar un aprendizaje significativo en estudiantes de nivel superior y de posgrado.

En la investigación llevada a cabo por González, Tovilla, Juárez y López (2017), sobre el uso de tecnologías de la información en el rendimiento académico basados en una población mexicana de estudiantes de Medicina, analizaron las calificaciones obtenidas en la asignatura de genética en estudiantes de la Licenciatura en Médico Cirujano de la División Académica Multidisciplinaria de Comalcalco, de la Universidad Juárez Autónoma de Tabasco. Para este propósito, se examinó el promedio de calificación obtenido por los estudiantes en tres periodos escolares. Para facilitar la comparación entre los diferentes periodos escolares, se agruparon las poblaciones de estos tres periodos en dos conjuntos: Los que utilizaron las herramientas tecnológicas de los Grupos de Google y los que no utilizaron esta herramienta virtual. Es importante mencionar que, en los tres periodos escolares, fue el mismo docente quien impartió la asignatura de genética. Concluyeron que resulta evidente el impacto positivo que la implementación de la herramienta virtual de Grupos de Google tuvo en el rendimiento académico de los estudiantes de Medicina, lo que se refleja de manera directa en su calificación promedio. Por lo tanto, sus resultados demostraron de modo fehaciente, que el uso de las tecnologías conlleva a un cambio en la dimensión gnoseológica de la actividad educativa, cambio que aún adolece de una base metodológica acabada. Estos resultados obtenidos en el estudio, sugieren que el uso de Tecnologías de la Información y Comunicación en la enseñanza de las Ciencias Médicas, como las herramientas virtuales de los Grupos de
Google, incremente el rendimiento académico en estos estudiantes, lo que se traduce en una mejor calificación promedio, González et al (2017).

En otras investigaciones, se enfatiza sobre el uso del foro virtual como estrategia de aprendizaje, se concluyó que favorece el desarrollo de pensamiento crítico en los estudiantes; tal como lo demuestra Mendoza, Mariel, Gómez, y García, (2016), quienes investigaron sobre el debate en línea mediante foros virtuales en plataformas educativas, como una herramienta para promover el pensamiento crítico. El experimento se realizó con estudiantes de segundo grado de secundaria en México. Se utilizó un diseño cuantitativo, mediante un pretest $\mathrm{y}$ un postest para la intervención de actividades y discusión en línea en la plataforma Moodle. El instrumento se utilizó para medir cinco indicadores de pensamiento crítico de acuerdo a lo consultado en la literatura: interpretación, análisis, inferencia, explicación y deducción. Se realizaron una serie de actividades en conjunto con la participación en foros, después se aplicó la segunda prueba $y$ se analizaron estadísticamente los resultados. Los hallazgos mostraron un incremento en las habilidades de raciocinio crítico después de la interacción en los foros. Se obtuvieron también las calificaciones individuales, en las que se evidencio una importante mejora en el desempeño de la deducción y explicación, mientras que la habilidad de menos aumento fue la inferencia. (Mendoza et al. 2015).

En concordancia con el tema de la presente investigación sobre el efecto de entornos virtuales y dentro de ellos el uso del foro virtual, encontramos también trabajos como el de Borgobello, Majul, y De Seta, (2019), quienes realizaron un análisis cualitativo de una propuesta pedagógica para 
la incorporación de entornos virtuales a la enseñanza universitaria en Argentina a partir de tres ejes: experiencia, rol docente y tiempo, encontrando que los entornos virtuales basados en el concepto de aprendizaje colaborativo generan oportunidades de diálogo que ponen en juego el conocimiento como una construcción colectiva. Este concepto de aprendizaje colaborativo, dada la importancia que tiene específicamente en relación a Moodle, fue uno de los ejes que se trabajaron en el taller, destacándose por parte de los participantes la importancia del intercambio de ideas, conceptos y experiencias.

\section{CONCLUSIONES}

De acuerdo con la revisión de la literatura, el conocimiento y difusión de las TICs es crucial para reestructurar la planificación y estrategia docente, en busca de optimizar el logro de competencias. Queda establecida la importancia y el impacto positivo de los foros de discusión como herramienta para mejorar el proceso de enseñanza-aprendizaje, su utilidad para la discusión de contenidos y para implantar un proceso de aprendizaje activo y participativo de los estudiantes. El desarrollo de habilidades inferenciales, pensamiento $\mathrm{y}$ juicio crítico son elementos adicionales trascendentes para la evolución del proceso educativo, mientras los estudiantes se convierten en creadores de las asignaturas, recogen y contextualizan la información acumulada en las plataformas habilitadas para tales fines, participan de manera activa $y$ aportan valor pedagógico al proceso educativo

En esta línea, con los argumentos ofrecidos en la literatura acerca de los foros virtuales, en especial los de debate $y$ argumentación, mejoran el aprendizaje del estudiante y favorecen la obtención de unos resultados académicos superiores, ya que facilitan y potencian el intercambio y la transmisión de información y la comunicación entre los miembros involucrados en el proceso.

En contraste con la formación tradicional, el estudiante que se desenvuelve bajo estas nuevas tecnologías, no experimenta una transición traumática del centro de estudios hacia una sociedad cada vez más exigente, pudiendo adaptarse a ella e incorporarse a sus procesos y oportunidades.

Una manifestación del aseguramiento de la calidad universitaria está dada por los procesos de revisión del currículo, los cuales pueden someterse a un proceso reflexivo de actualización y adecuación requeridas por las necesidades de mejorar el control, incluir cambios producidos en la disciplina profesional, y finalmente incluir cambios en las estrategias de enseñanza como la incorporación de foros virtuales de debate y argumentación.

\section{REFERENCIAS}

Benavides, R., Villacís, M., y Ramos, J. (2017). El Entorno Virtual de Aprendizaje (EVA) en la Generación de Conocimiento de Estudiantes Universitarios CienciAmérica. Revista de Divulgación Científica de La Universidad Tecnológica Indoámerica

Borgobello, A., Majul, S., y De Seta, D. (2019). Análisis cualitativo de una propuesta pedagógica para la Incorporación de entornos virtuales a la enseñanza universitaria en Argentina a partir de Tres Ejes: Experiencia, Rol Docente y Tiempo.Instituto Rosario de Investigaciones en Ciencias de la Educ. Hamut'ay, 61-97. doi:http://dx.doi.org/10.21503/hamu.v6i3 .1848 
Castro, N., Suárez, X., y Soto, V. (2016). El uso del foro virtual para desarrollar el aprendizaje autorregulado de los estudiantes universitarios. . Innovación educativa (México, DF), 16(70), 23-41

Díaz, R. (2018). Conocimientos, actitudes y prácticas sobre el uso de redes sociales digitales y su rol frente a la salud $y$ socialización de los estudiantes de Medicina de la Pontificia Universidad Católica del Ecuador en el período septiembre noviembre 2018. Quito, Ecuador: Trabajo especial de grado de la Pontificia Universidad Católica del Ecuador para optar al título de Médico Cirujano. Obtenido de http://repositorio.puce.edu.ec/handle/22 $000 / 16236$

Galindo, V., De la Torre, C., y Vera, H. (2020). Influencia del uso de un entorno virtual en el rendimiento académico de estudiantes de Odontología. [Tesis de doctorado, Universidad Nacional de San Antonio Abad del Cusco]. http://revistas.unsaac.edu.pe/index.

Revista SITUA, 23(1), 27-34. Obtenido de http://revistas.unsaac.edu.pe/index.php/S ITUA/article/view/209

González, K., Tovilla, C., Juárez, I., y López, M. (2017). Uso de tecnologías de la información en el rendimiento académico basados en una población mexicana de estudiantes de Medicina. Educ Med Super 31(2), 1-10. Obtenido de http://scielo.sld.cu/scielo.php?script=sci_a rttext\&pid=S0864-

$21412017000200008 \& \operatorname{lng}=e s \& t \operatorname{lng}=e s$

Guzmán, Y., y Flores, R. (2020). La competencia argumentativa como meta en contextos educativos. Revisión de la literatura. Educar.

doi:https://doi.org/10.5565/rev/educar.1 009

Icarte, G., y Labate, H. (2016). Metodología para revisar y actualizar un diseño curricular de una carrera universitaria que incorpora el concepto de aprendizaje basado en competencias. Formación universitaria, $\quad 9 \quad$ (2), 3-16. doi:https://dx.doi.org/10.4067/S071850062016000200002

Kutugata, A. (2016). Foros de discusión: herramienta para incrementar el pensamiento crítico en educación superior. Apertura 8(2), 84-99. doi:http://dx.doi.org/10.18381/Ap.v8n2.8 80

La Madriz, J. (2016). Factores que promueven la deserción del aula virtual. . Orbis. Revista Científica Ciencias Humanas, 12(35), 18-40

Mendoza, E., Mariel, I., Gómez, M., y García, I. (2016). El desarrollo del pensamiento crítico mediante el debate asincrónico en foros virtuales en educación secundaria. Aletheia, 8(1), 100-115. Obtenido de http://www.scielo.org.co/scielo.php?script =sci_arttext\&pid=S2145-

Merchán, M., Saavedra, F., García, J., Ospina, N., Aragón, C., y Martínez, J. (2017). Estudio de casos y controles de factores relacionados con el rendimiento académico en estudiantes de medicina. Educación Médica Superior 31, 101-109. Obtenido de http://scielo.sld.cuhttp://scielo.sld.cu

MINEDU. (2020). Resolución viceministerial, $N^{\circ}$ 00095-2020. Perú: Informe del Ministerio de Educación. Obtenido de http://esinad.minedu.gob.pe/e_sinadmed_ 5/VDD_ConsultaDocumento.aspx

Moncayo, N., Pereira, J., y Luján, M. (2018). Las actividades de aprendizaje y el rendimiento académico en la educación a distancia. Universidad Técnica Particular de Loja, Ecuador. Revista UNIMAR. doi:https://doi.org/10.31948/unimar.361.2

Navarro, V., Cragno, A., y Di Bernardo, J. (2016). Analítica del Aprendizaje aplicada a los estudiantes de Medicina en Foros de discusión de casos clínicos en Medicina III. Universidad Nacional del Nordeste. Revista Argentina de Educación Médica. 7(1), 14$18 . \quad$ Obtenido de https://www.researchgate.net/publication /330195920 
Ordaz, A., y García, O. (2018). El estudio del rendimiento académico en nivel universitario. Aproximaciones al estado del conocimiento. . VIII CLABES Octava Conferencia Latinoamericana Sobre El Abandono En La Educación Superior

Palella, S., y Martins, F. (2010). Metodología de la investigación cuantitativa. Caracas, Venezuela: FEDUPEL, Fondo Editorial de la Universidad Pedagógica Experimental Libertador

Soto, J., Torres, C., y Zamudio, J. (2018). Valoraciones acerca de la colaboración en línea en estudiantes de posgrado en servicios de salud. Apertura (Guadalajara, Jal.) 10(2), 64-79. doi:https://doi.org/10.18381/ap.vl0n2.13 70
USS. (2014). Guía del estudiante. Chiclayo, Perú: USS Virtual. Obtenido de http://www.ussvirtual.edu.pe/descargas/ PEaD/GUIA_DEL_ESTUDIANTE_2013.pdf

Villalobos, M. (2018). Efecto de las aulas virtuales en el aprendizaje por competencias en los estudiantes del Instituto Superior Cueto Fernandini, Comas. Tesis de maestría, Universidad Nacional De Educación Enrique Guzmán y Valle. Obtenido de http://repositorio.une.edu.pe/handle/UNE $/ 2355$ 